ability to cause acute flaccid myelitis. Acute flaccid myelitis (AFM) refers to acute flaccid limb weakness with predominantly gray matter spinal cord lesions on imaging or evidence of anterior horn cell damage on electrodiagnostic studies. In 2014 , in the midst of a large outbreak of EV D68-associated respiratory disease in the United States, clusters of AFM were noted in children. Enhanced nationwide surveillance ultimately identified 120 cases of AFM. Enterovirus D68 was the most common virus detected in these patients. Full recovery was rare (5\%). More recently, a 2019 report describes 29-cases of EV D68-associated AFM diagnosed in 12 European countries in 2016. We present the first cases of EV D68-associated AFM in Ireland to date.

Clinical cases In June 2016, a previously well 2-year 9 month old girl presented with a one-week history of cough, fever and vomiting. Thirty-six hours later, respiratory status worsened and she developed AFM and bulbar palsy necessitating intubation and ventilation. CSF analysis demonstrated a lymphocytic pleocytosis. In October 2018, a 4-year old boy, with a background of acute lymphoblastic leukaemia diagnosed 17 months previously, presented with fever, worsening cough and pancytopenia. Three-days later, he required intubation and ventilation after a respiratory arrest. On weaning sedation, eight-days later, he displayed clinical signs of AFM. Both children had nasopharyngeal aspirates (NPA) PCR positive for rhinovirus/enterovirus, and stool/rectal swabs PCR positive for EV. CSF was PCR negative for EV, HPE, HSV, HHV-6, VZV and adenovirus. MRI showed extensive abnormal signal predominantly of white matter in the cervico-thoracic cord. Electromyography was indicative of anterior horn cell involvement. Both children required prolonged ICU and hospital stays, have severe residual neurologic deficits with flaccid quadriparesis, tracheostomy and require ongoing ventilatory support.

Results Subsequent subtyping of EV isolates from both children was positive for EV D68. Additional testing of 800 rhinovirus/enterovirus-positive NPA specimens submitted to the National Virus Reference Laboratory (NVRL) from August to October 2018 detected 30 (3.75\%) EV D68-positives.

Conclusion This report of two-children with rapidly progressive AFM following prodromal febrile respiratory or gastrointestinal illness in association with EV D68 infection and recent results of enhanced European and local surveillance suggests that EV D68 AFM has arrived in Ireland.

\section{GP199 CELL RATIOS IN TRAUMATIC CEREBROSPINAL FLUID. DO THEY HAVE PREDICTIVE VALUE FOR MENINGITIS?}

Ali Raba*, Jean Donnelly. Our Lady's Children's Hospital, Crumlin, Dublin, Ireland

\subsection{6/archdischild-2019-epa.259}

Background and aims A cerebrospinal fluid (CSF) red to white cell correction ratio of $500: 1$ is often quoted as a preliminary result for determining potential positive/negative CSF cultures. The aim of this study is to examine the diagnostic utility of correcting white blood cells (WBC) counts in CSF based on 500:1 ratio and to evaluate this adjustment in identifying bacterial meningitis prior to final culture in infants.

Method We performed a cohort study on children younger than 3 months who admitted to Our Lady's Children's Hospital, Crumlin, with suspected sepsis from the $1^{\text {st }}$ October 2017 to the $05^{\text {th }}$ October 2018. CSF WBC counts were adjusted downward for traumatic lumbar punctures (RBCs > $500 / \mathrm{mm} 3)$ using white to red cells $(500: 1)$ ratio. We calculated sensitivity, specificity, positive productive value (PPV) and negative productive value (NPV) of unadjusted and adjusted WBC count in traumatic CSF for predicting bacterial meningitis.

Result A total of 117 infants (age: $39.4 \pm 21.7$ days, male: 85 (72.6\%), traumatic lumber puncture: $35(29.9 \%)$ ) were enrolled in this study. Among 117 infants, 3 (2.6\%) had positive CSF cultures for bacterial meningitis, 28 (23.9\%) had viral meningitis. Compared with the unadjusted cerebrospinal fluid WBC count, the adjusted one had similar sensitivity, PPV and NPV for bacterial meningitis. NPV and PPV of adjusted CSF WBC count were $100 \%$ and $14.2 \%$, respectively (sensitivity $100 \%$, specificity $82 \%$ ). While NPV and PPV of unadjusted one were $100 \%$ and $5.8 \%$, respectively (sensitivity 100\%, specificity $52 \%)$.

Conclusion Adjusted WBC counts in CSF have no advantage over uncorrected counts for predicting bacterial meningitis. All available clinical and laboratory data should be used when deciding whether to institute treatment in an infant with suspected meningitis and bloody CSF.

\section{GP200 IS THE INFLUENZA IN NEONATES A DANGEROUS INFECTION? - OWN OBSERVATIONS}

1,2Edyta Zawłocka*, 1,2 Małgorzata Czajkowska, 1,2 August Wrotek, 1,2Teresa Jackowska. ${ }^{1}$ Department of Pediatrics, the Medical Centre of Postgraduate Education, Marymoncka 99/ 103, Warsaw, Poland; '2Department of Pediatrics, Bielanski Hospital, Cegłowska 80, Warsaw, Poland

\subsection{6/archdischild-2019-epa.260}

Background Influenza is a viral infectious disease affecting every age group, including neonates and infants.

Aim To analyze the frequency, clinical signs and course of influenza in neonates hospitalized during three influenza epidemic seasons.

Material and Methods 12 neonates were hospitalized due to influenza in Department of Pediatrics in three influenza epidemic seasons. Rapid Influenza Diagnostic Tests (RIDTs) and/ or Real Time-Polymerase Chains Reaction Tests (RT-PCR) were performed in patients with a clinical suspicion of influenza.

Results From September 2015 to August 2018 we hospitalized 294 patients with influenza. The youngest patient was 12 days old, the oldest - 17 years 3 months. 4.1\% (12/294) were neonates. The most common reason for hospital referral was fever $(58.3 \%$; 7/12). Other observe symptoms were: coryza $(58.3 \% ; 7 / 12)$, change of behavior $(58.3 \% ; 7 / 12)$, cough $(50 \% ; 6 / 12)$, dyspnea $(40 \% ; 5 / 12)$ and apneas $(8.3 \% ; 1 / 12)$. RIDTs were performed in seven $(58.3 \%)$ of neonates, but positive results were only in 2 cases (16\%). The final diagnosis was made using RT-PCR tests. $83.3 \%(10 / 12)$ of neonates were diagnosed with influenza A, and 1.7\% (2/12) influenza B. Complications occurred in eight neonates. $75 \%(9 / 12)$ patients had contact with the infection at home. None of the families were influenza-vaccinated before the season. The average time of hospitalization was 10.2 days (from 6 to 15 days).

Conclusion The course of influenza in neonates can be severe. RIDTs seem not very useful in diagnose.

This study was supported by CMKP. Grant number 5011--20-19-18 